\title{
Forbidden Traffic Signs Detection and Recognition Based on Sparse Representation
}

\author{
Sheng Guo \\ School of Information and Communication Engineering \\ Dalian University of Technology \\ Dalian, China \\ e-mail:guosheng-14@163.com
}

\author{
Jianhua Li \\ School of Information and Communication Engineering. \\ Dalian University of Technology \\ Dalian, China \\ e-mail:jianhual@dlut.edu.cn
}

\begin{abstract}
This paper presents an automatic traffic-sign detection and recognition algorithm based on sparse representation. Our system consists of several steps. In the first stage, we detect potential traffic signs using the most remarkable feature-color-extracted by HSI model, which is immune to lighting changes. In the second stage, the preprocessing stage, image binaryzation and image cutting help the system to extract candidate traffic-sign regions. Then OMP algorithm is performed to calculate the sparse coefficients of candidate traffic-sign regions on dictionary $D$. The dictionary $D$ is constructed by training images with different rotation. Finally, the sparse coefficients are used to classify. The algorithm proposed offers high performance and better accuracy especially in variable lighting conditions and rotation. Because of its real time and accuracy, this algorithm can be used in real world application.
\end{abstract}

Keywords-Detection; HSI; recognition; forbidden traffic signs; sparse representation.

\section{INTRODUCTION}

Traffic signs detection and recognition is becoming more and more important in intelligence traffic system recently. Few drivers can balance focusing on traffic condition and noticing important traffic signs, especially in rush hours. This field can make drivers pay more attention to traffic condition by informing drivers traffic signs automatically.

In the real world application, the images we are going to deal with are taken in a moving car, so some problems are unavoidable. The first problem is how to overcome the influence of variable lighting condition. We hope that after processing, the influence of lighting can be decreased or even eliminated. Another problem is the rotation of the signs. Traffic-signs images taken in real world may have rotation more or less. This will influence the performance of detection on traffic signs. At last, occlusions caused by trees or dirt on the traffic signs may also reduce the rate of recognition. Considering the disadvantages above, our algorithm should be invariant to the rotation and occlusions.

The traffic signs can be classified into three basic classes generally. They are forbidden signs, warning signs

\author{
Shuping Zhao \\ Dept.of Information Engineering \\ Dalian Ocean University \\ Dalian, China \\ e-mail:zsp_60@126.com
}

and mandatory signs, and some of them are shown in Fig.1. In these three classes, forbidden traffic signs' information is the most urgent, such as speed limitation, no entry for specific vehicles and so on. So in this paper, we mainly focus on detection and recognition of forbidden traffic signs, the other two kinds of traffic signs have the same process and similar results.

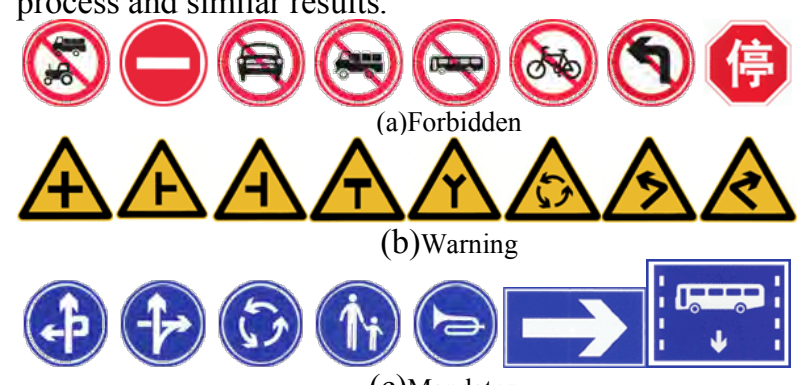

(c)Mandatory

Figure 1. Three different kinds of traffic signs

The organization of this paper is given as follows: Section II will introduce previous work in this field, both in detection and recognition. In Section III, which is the most important part of our paper, we will describe our algorithm in detail. Results illustrated in Section IV will confirm the robustness of the proposed method. Finally, conclusion will be presented in Section V.

\section{RELATED WORK}

Sensibly, the algorithm proposed can be divided into two main stage: (1)detection and (2)recognition. A significant number of papers introducing different methods in each step have been published.

In detection stage, our goal is to find interesting area or locate some potential traffic signs. Algorithm based on color and edge is widely used because traffic signs are designed to be remarkable to the background.

In [1], a ratio between red component and the sum of RGB components is used to detect red pixels. Although the RGB model decomposition is one of original algorithms used to detect, it is more easily to be influenced. Now, HSI[3], YUV[2] and CIECAM97[4] are the most popular 
way to detect potential interesting area. In [5], proper thresholds on hue and saturation bands are fixed to extract the certain color. In [6], a nonlinear transformation over hue and saturation is employed to enhance the desired colors in the image (red and blue) using two look-up tables for every color for which we are looking.

Some other traffic sign detection algorithms are based on edge detection. In [7], Hough transformation(HT), a classic circle detection and line detection way, has a relatively good performance despite of the time-consuming processing. In recent work, some new methods are based on radial symmetry[8]. With the help of gradient image and the radius set previously, a circle or a line with certain radius and center is located. The main advantage of this method is that it is able to run in real time, but occlusion has great impact on the performance.

Several special methods are applied in detection, such as genetic algorithm(GA)[9] and template matching [10].

Once the potential signs are detected, after several preprocessing, they are sent to the next stage, recognition. Neural networks(NN)[12] is the most commonly-used method, the NN system can reach to a high rate of recognition. But the poor real-time and its fixed architecture limit the flexibility of application. Based on $\mathrm{NN}$, a committee of convolutional neural networks (CCNNs) [11] is used to classify various traffic signs. In [13], HU invariant moment over support vector machine(SVM) shows that it has better effect in computation complexity, and $\mathrm{HU}$ invariant moment is invariant to rotation. K-Nearest Neighbor(KNN)[17] calculates the Euclidean distance between the test images and the trained template images, then decide which class the sample images belong to. Some other useful features are performed in recognition stage, such as HOG [16], DtB, Fourier descriptors[15] and PCA[14].

\section{TRAFFIC SIGN DETECTION AND RECOGNITION}

\section{A. Overview of the algorithm}

In this paper, we present an algorithm for detection and recognition of traffic signs that has been successfully applied to Chinese traffic signs. The whole system consists of three stages, as shown in Fig.2.

- Detection: Red pixels are detected by thresholds using HSI model to reduce the influence of lighting. The aspect ratio of a connected component is used to determine whether it will be a candidate traffic-sign regions.

- Preprocessing: In this stage, available region or inside region of a candidate traffic-sign is extracted by removing the border, binaryzation, and imagesize normalization, and so on.

- Recognition: OMP is used to compute the sparse coefficients of the available region on dictionary $\mathrm{D}$. The dictionary $\mathrm{D}$ is constructed by training images with different rotation. Finally, the sparse coefficients are used to classify.

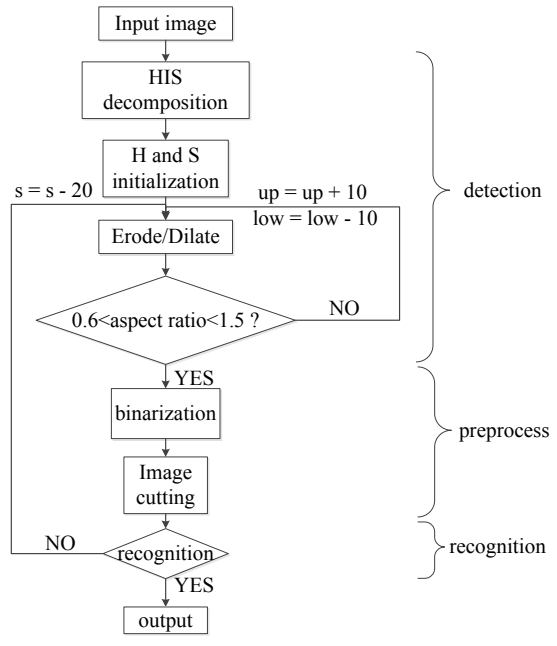

Figure 2. Flowchart

\section{B. Detection}

HSI color model is immune to the lighting changes, and most color information consists in saturation component and hue component, so it is usually used in real-time traffic sign detection.

In this paper, we detect red pixels of test image to extract candidate traffic-sign regions. We set upper bound of hue to be 30 , and lower bound to be 210 in initialization, which means that hue component within 0 to 30 and 210 to 255 is considered. Saturation component is set 100 originally. Both hue and saturation component can be changed in the whole system to get adapted to the natural scene. As shown in Fig.3, pixels that meet both the hue and saturation requirements in the original image are set to white in the labeled image, Fig.3(b). Then the aspect ratio of a connected component is used to eliminate noises and obtain final candidate regions. Of course, we still can' t avoid that some regions meet all requirements we set, just like Fig.3(c) and Fig.3(d). It will be sent to preprocessing and recognition step, and we will talk about it later.

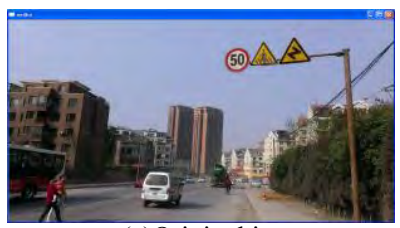

(a)Original image

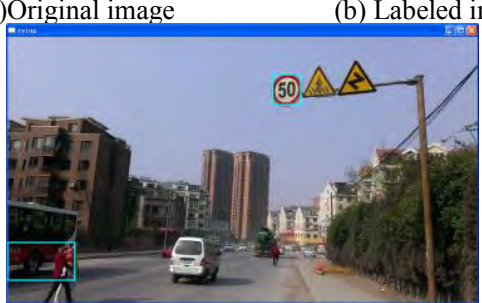

(c) Rectangle in original image

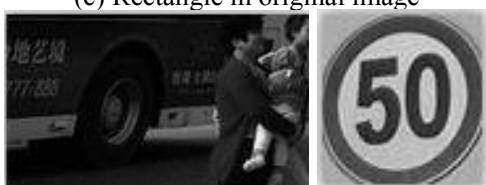

(d) Image detected

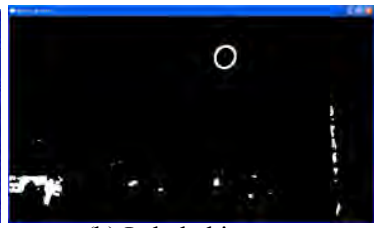

\section{(1)}




\section{Preprocessing}

All candidate regions extracted in Detection stage will be done preprocessing operation shown as follow.

The sizes of signs in images are different because of the varied distance between the traffic sign and a moving car. To process regions of interest uniformly, we resize them to $128 * 128$. It is clearly found from Fig. 1 that content inside the circle carry the most information that drivers should notice, so we discard the outer fringe directly just like Fig.4. Despite of the immunity brought by HSI color model, traffic signs are still affect by lighting changes, as shown in Fig.5. OTSU, a classical method to change a gray image into a binary image, is performed to eliminate the influence caused by lighting. Then all pixels whose distance to the center of the image is lager than a certain threshold will set to black, so more noises are deleted in Fig.4. Some pixels remain exist caused by random rotation and occlusion. Calculate the area of connected component and discard some of them if it is lower than threshold. Minimum enclosing rectangle helps us present the information maximally in Fig.4. At last, we change the size of the image into $64 * 64$ waiting to be sent to the recognition stage.

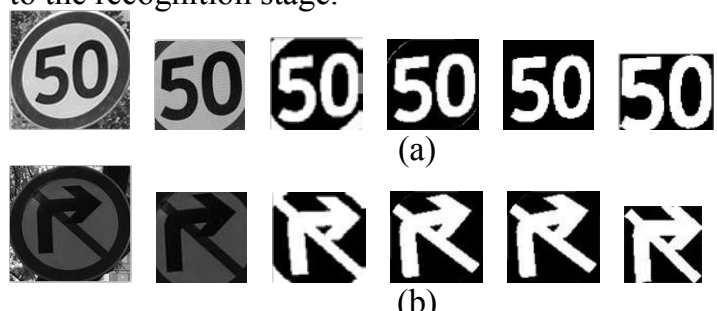

Fig 4. Examples of preprocessing stage
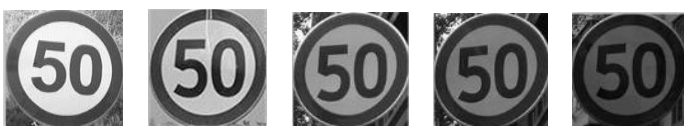

Fig 5. Affected by lighting changes

\section{Recognition}

Inspired by [18], we first talk about sparse code(SC).

Given sufficient training samples of the $i$ th object class, $\mathbf{D}_{\mathrm{i}}=\left[\mathbf{d}_{i, 1}, \mathbf{d}_{i, 2}, \ldots, \mathbf{d}_{i, n_{i}}\right] \in \mathbf{R}^{\mathrm{m}^{*} n_{i}}$, any new sample $\mathbf{y} \in R^{m}$ from $\boldsymbol{i}$ th class will approximately lie in the linear span of the training samples:

$$
\mathbf{y}=\alpha_{i, 1} * \mathbf{d}_{\mathbf{i}, \mathbf{1}}+\alpha_{i, 2} * \mathbf{d}_{\mathbf{i}, \mathbf{2}}+\ldots+\alpha_{i, n_{i}} * \mathbf{d}_{\mathbf{i}, \mathbf{n}_{\mathbf{i}}}
$$

where $\alpha_{\mathrm{i}, \mathrm{j}} \in R, j=1,2, \ldots, n_{i}$.

We define a new matrix D, shown in Fig.6, for the entire training set as the concatenation of the $\mathrm{n}$ training samples of all k object classes:

$$
D=\left[D_{1}, D_{2}, \ldots, D_{K}\right]=\left[v_{1,1}, v_{1,2}, \ldots, v_{k, n_{k}}\right]
$$

Then, the linear representation of $y$ can be rewritten in terms of all training samples as

$$
\mathbf{y}=\mathbf{D} \boldsymbol{\alpha}
$$

where $\quad \boldsymbol{\alpha}=\left[0, \ldots, 0, \alpha_{i, 1}, \alpha_{i, 1}, \ldots, \alpha_{i, 1}, 0, \ldots, 0\right]^{T} \in R^{n} \quad$ is $\quad$ a coefficient vector whose entries are zero except those associated with the $i$ th class.

However, the solution $\alpha$ of (3) is generally dense, with large nonzero entries corresponding to training samples from many different classes. This motivates us to seek the sparsest solution to $\mathbf{y}=\mathbf{D} \boldsymbol{\alpha}$, solving the following optimization problem:

$$
\hat{\boldsymbol{\alpha}}=\arg \min \|\boldsymbol{\alpha}\|_{0} \quad \text { subject to } \quad \mathbf{y}=\mathbf{D \alpha}
$$

where $\|.\|_{0}$ denotes the $L_{0}$-norm, which counts the number of nonzero entries in a vector. If the solution $\alpha$ is sparse enough, (4) can be easily solved following $L_{1}$-norm problem:

$$
\hat{\boldsymbol{\alpha}}=\operatorname{argmin}\|\boldsymbol{\alpha}\|_{1} \quad \text { subject to } \quad \mathbf{y}=\mathbf{D} \boldsymbol{\alpha}
$$

Given a new test sample $y$ from one of the classes in the training set, after we compute its sparse representation $\boldsymbol{\alpha}$, the locations of nonzero entries in $\boldsymbol{\alpha}$ belong to class $\boldsymbol{i}$, and we can easily assign the test sample $\mathrm{y}$ to that class. However, noise may lead to small nonzero entries, we can easily assign $y$ to the class with the single largest entry in $\boldsymbol{\alpha}$. If the test sample is a noise region, the entries of sparse coefficients are more flat. The situations above are the main bases in recognition stage.

Assume that we have dictionary D and a test sample, Orthogonal Matching Pursuit(OMP) is one of the most efficient algorithms used to get sparse coefficients.

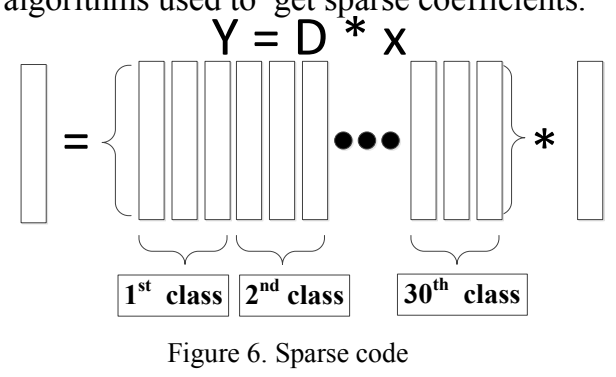

From the SC algorithm mentioned above, the key of the algorithm is building a dictionary containing all kinds of situation in real world, including lighting changes and rotation. Because of the binaryzation in the last stage, influences by lighting changes are almost eliminated. Therefore, rotation is our priority in selection of atoms.

Briefly described in Fig.6, every class has 10 training images containing possible levels rotation, as shown in Fig.7. We reshape $64 * 64$ images sent by last stage into $64^{2} * 1$ column vector. 10 columns consist into one class, and 30 classes consist into D. After normalizing, we get the final dictionary with size of $64^{2 *}(10 * 30)$.

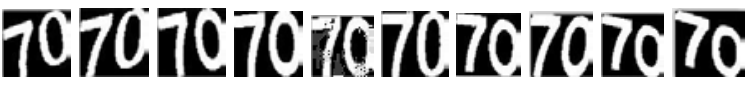

Figure 7. Different levels of rotation in D

Following the same way, new test image is reshaped into $64^{2} * 1$ normalized column vector. OMP is performed to solve $\boldsymbol{\alpha}$ in (3). We set sparsity 10 , it means that sparse coefficients $\boldsymbol{\alpha}_{300^{*} 1}$ just have 10 nonzero coefficients. By experiments, we find that we can get better results when the sparsity is 10 , more than 10 means higher recognition rate but consuming longer time, less than 10 means consuming shorter time but lower recognition rate. One of sparse coefficient vectors is shown in Table.1. Clearly, 
gamma $(61,1)$ has the peak value, and sum from $\operatorname{gamma}(61,1)$ to $\operatorname{gamma}(70,1)$ is larger than any value of other classes.

All sparse coefficients contribute to classifying by adding corresponding absolute number of coefficients in each class, such as $1 \sim 10,21 \sim 30$ and so on. At last, the identifying image belongs to the class whose sum has the largest value.

TABLE I. A EXAMPLE OF SPARSE COEFFICIENT VECTOR

\begin{tabular}{|c|c|}
\hline Location & Value \\
\hline 6 & 0.0631 \\
\hline 36 & -0.1714 \\
\hline 37 & 0.1988 \\
\hline 61 & 0.4404 \\
\hline 64 & -0.0749 \\
\hline 68 & 0.2469 \\
\hline 70 & 0.0799 \\
\hline 71 & 0.2305 \\
\hline 150 & 0.0408 \\
\hline 158 & -0.0506 \\
\hline
\end{tabular}

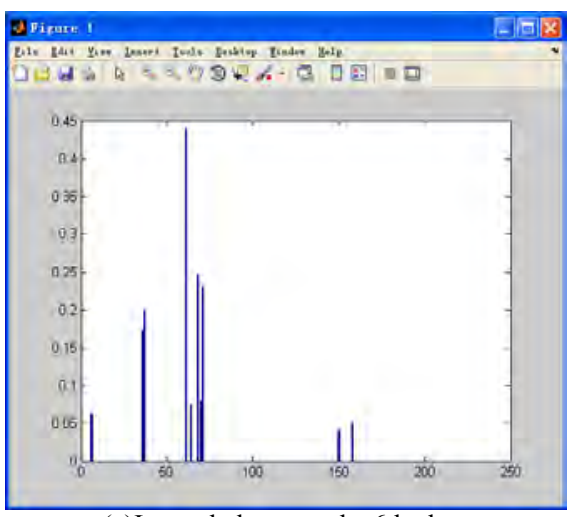

(a)Image belongs to the 6th class

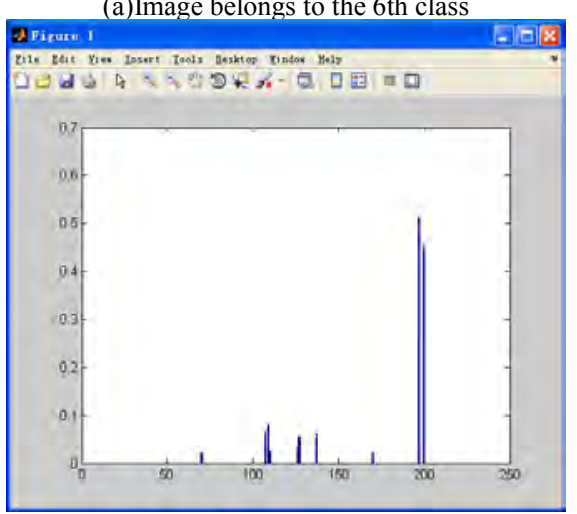

(b)Image belongs to the 20th class

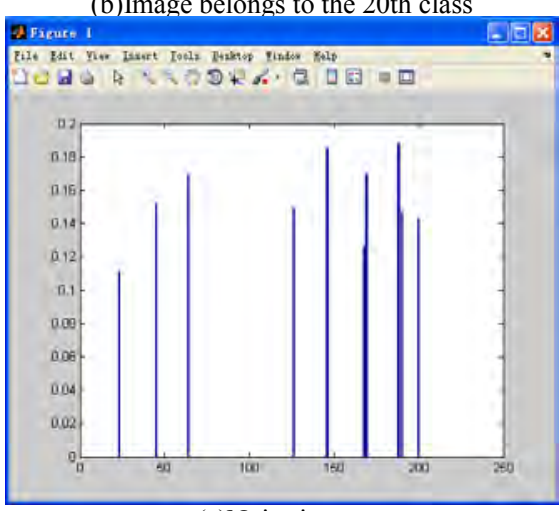

(c)Noise image

Figure 8. Histograms of coefficients' absolute value
In a real world application, we can't be sure that test images sent by detection stage are traffic signs, so some noise regions which meet the requirements are also sent to the recognition stage, just like people in red in Fig.3(d). The variance in statistics can help us to solve the problem. Fig. 8 shows histograms of coefficients' absolute value, it shows that coefficients of images belonging to some class have at least one clear peak. However, sparse coefficients of a connected component which may be not traffic signs are more flat. Variance can reflect the volatility of a set of data, so the algorithm proposed decides whether an image is a traffic sign or not: if the variance is higher than threshold, test image is a traffic sign, or it is not a traffic sign.

\section{RESULTS AND DISCUSSION}

In this paper, we fix the camera(android phone) on the window of a moving car at different speed. To test the feasibility of our proposed algorithm, 10000 images of about 30 classes taken from android phone under various weather conditions, at different times of a day are used. The images which are in JPG format and 1000*530 pixels are run in MATLAB platform installed on a computer with 3.30GHz Intel Core i3 CPU.

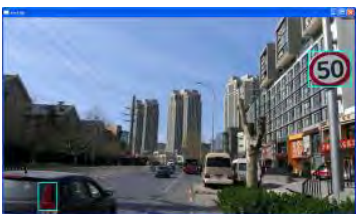

(a)Noise in an image

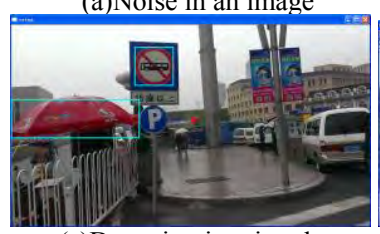

(c)Detection in rainy day

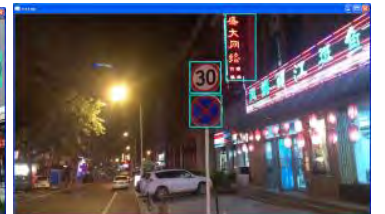

(b)Detection at dark

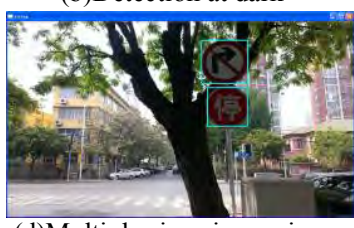

(d)Multiple signs in one image

Fig 9. Experiment results in different conditions

Fig.9 shows detection experiment results in different conditions. As mentioned in INTRODUCTION, the algorithm can be adapted to all kinds of conditions, including sunny, Fig.9(a), foggy and rainy day, Fig.9(c), and it should also have a good performance at night, Fig.9(b). In addition, multiple signs in one image, Fig.9(d), is quite common in real world. During application, noise candidate blobs can not be avoided, especially at night, when there are more red lights than in the daytime.

In recognition stage, the sparsity $\mathrm{k}$ plays an important role in recognition. There is direct relationship between time consumption and $\mathrm{k}$, and so does rate of recognition. Several classes are select randomly to choose appropriate $\mathrm{k}$, we change $\mathrm{k}$, and observe performance of algorithm. Finally, we select an appropriate $\mathrm{k}$ and fix it.

From Table.2, we just mainly focus on the $\mathrm{k}$ changing between 4 to 18 . We can conclude that with sparsity $\mathrm{k}$ increasing, the algorithm has higher rate of recognition but consumes longer time. When $\mathrm{k}$ is larger than a certain threshold, $\mathrm{k}$ is 12 in this experiment, the growth rate of average recognition rate becomes slower. Though recognition rate is prior in the algorithm proposed, time consumption is still a significant impact factor in our system. From Table.2, it is clear that the sparsity k can lead 
to unlimited increasing of time consumption. To balance recognition rate and time consumption, we set sparsity $\mathrm{k}$ 10, which makes our system has a relatively high recognition rate while consuming less time.

TABLE II. RELATIONSHIP BETWEEN SPARSITy, TIME AND RECOGNITION RATE

\begin{tabular}{|c|c|c|c|c|c|c|c|c|}
\hline $\mathbf{k}$ & 4 & 6 & 8 & 10 & 12 & 14 & 16 & 18 \\
\hline $\mathrm{t}$ & 0.400 & 0.543 & 0.685 & 0.843 & 0.987 & 1.142 & 1.284 & 1.443 \\
\hline $\mathrm{r}$ & 82.15 & 90.48 & 93.42 & 95.49 & 96.51 & 97.12 & 97.20 & 97.25 \\
\hline
\end{tabular}

\begin{tabular}{|c|c|c|c|c|c|}
\hline & \multicolumn{5}{c|}{ RATE OF RECOGNITION IN DIFFERENT CONDITION } \\
\hline & Sunny & $\begin{array}{c}\text { Foggy } \\
\text { Rainy }\end{array}$ & At night & Total & $\begin{array}{c}\text { Noise } \\
\text { Candidate } \\
\text { images }\end{array}$ \\
\hline total & 7046 & 959 & 1927 & 9932 & 469 \\
\hline $\begin{array}{c}\text { Correctly } \\
\text { classify }\end{array}$ & 6809 & 918 & 1768 & 9495 & 461 \\
\hline Rate & 96.6 & 95.7 & 91.7 & 95.6 & 98.2 \\
\hline
\end{tabular}

We set $\mathrm{k}=10$, and verify that it is still reasonable in other classes shown in Fig.10. There are about 10000 test images, and each class contains 400 images approximately, the results are shown in Table.3, as described in it, our

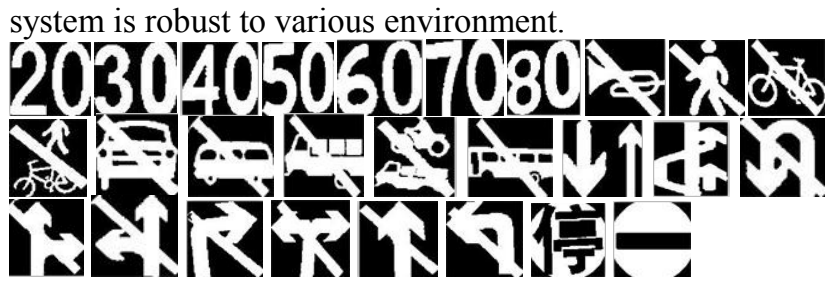

Figure 10. All kinds of traffic signs classes used in our paper

From Table.3, it shows that the recognition rate in rainy/foggy day is so close to it in sunny because of its robustness to lighting changes after some preprocessing stage. The rotation turns out to be the main consideration in selection of atoms. As a matter of fact, the angle of rotation is limited into 45 degree, which can be presented with 10 atoms. As for occlusion, small blobs have been discarded by connected component detection.

The biggest advantage of the algorithm is it can finish the classification using only one time calculation, unlike SVM in multi-class classification problem, or tree structure classification. If we want add a new class, just add atoms of this class into diction $\mathrm{D}$, without update the whole system, $\mathrm{NN}$ for example.

\section{CONCLUSION}

From PART IV, we can conclude that our algorithm perform well in real world. Using sparse presentation algorithm in recognition, our system is robust to lighting changes and rotation. The same processing can be used in warning traffic signs and mandatory traffic signs, just change arguments like hue component and add dictionaries. In the future, we can use some certain algorithms to reduce the dimension of an image, which can make recognition more time saving.

\section{ACKNOWLEDGMENT}

Identify applicable sponsor: This research was supported in part by Plan of Liaoning Science and
Technology Department of China (2010216008),Plan of Dalian Municipal Science and Technology Bureau of China (2011A16GX057).

\section{REFERENCES}

[1] H. Kamada, S. Naoi, and T. Gotoh, "A compact navigation system using image processing and fuzzy control," in Proc. Southeastcon, New Orleans, LA, Apr. 1990, vol. 1, pp. 337-342.

[2] J. Miura, T. Kanda, and Y. Shirai, "An active vision system for real-time traffic sign recognition," in Proc. IEEE Intell. Transp. Syst., Oct. 2000,pp. 52-57.

[3] A. de la Escalera, J. M. Armingol, J. M. Pastor, and F. J. Rodríguez, "Visual sign information extraction and identification by deformable models for intelligent vehicles," IEEE Trans. Intell. Transp. Syst., vol. 15,no. 2, pp. 57-68, Jun. 2004.

[4] X. Gao, L. Podladchikova, D. Shaposhnikov, K. Hong, and N. Shevtsova, "Recognition of traffic signs based on their color and shape features extracted using human vision models," J. Vis. Commun. Imag. Represent.,vol. 17, no. 4, pp. 675-685, Aug. 2006.

[5] S. Maldonado, S. Lafuente, P. Gil, H. G' omez, and F. L' opez, "Road-sign detection and recognition based on support vector machines" , IEEE Trans. on Intelligent Transportation Systems, Pending of publication, pp. 264-278, June 2007.

[6] A. de la Escalera, J. M. Armingol, J. M. Pastor, and F. J. Rodríguez, "Visual sign information extraction and identification by deformablemodels for intelligent vehicles," IEEE Trans. Intell. Transp. Syst., vol. 15,no. 2, pp. 57-68, Jun. 2004.

[7] Garda-Garrido M. A., Sotelo M. A., and Martfn-Gorostiza E., "Fast traffic sign detection and recognition under changing lighting conditions", Proc. IEEE Intelligent Transportation Systems Conference, pages 811-816, Toronto (Canada), 2006.

[8] G. Loy and N. Barnes, "Fast shape-based road sign detection for a driver assistance system," in International Conference on intelligent Robots and Systems, 2004

[9] Y. Aoyagi, T. Asakura, A study on traffic sign recognition in sceneimage using genetic algorithms and neural networks, 22nd International Conference on Industrial Electronics, Control, and Instrumentation, IEEE August (1996).

[10] HSU S.H., HUANG C.L.: "Road sign detection and recognition using matching pursuit method",Image Vis. Comput., 2001,19, pp $119-129$

[11] P. Sermanet and Y. LeCun, "Traffic sign recognition with multiscale convolutional networks," inProc. Int. Joint Conf. Neural Netw., 2011,pp. 2809-2813.

[12] F. Moutarde, A. Bargeton, A. Herbin, and A. Chanussot, "Robust onvehicle real-time visual detection of American and European speed limitsigns with a modular traffic signs recognition system," in Proc. IEEEIntell. Veh. Symp., 2007, pp. 1122-1126

[13] Fu Yan, Wang Mei, Zhang Chunqin. "SAR Image Target Recognition Based on $\mathrm{Hu}$ Invariant Moments and SVM". 2009 Fifth International Conference on Information Assurance and Security. 585-588

[14] S. Yan, D. Xu, B. Zhang, H. Zhang, Q. Yang, and S. Lin, “Graph Embedding and Extensions: A General Framework for Dimensionality Reduction," IEEE Trans. on Pattern Analysis and Machine Intelligence, vol. 29, no. 1, pp. 40-51, Jan. 2007.

[15] Larsson, F,Felsberg, M, o n -E.,"Correlating fourier descriptors of local patches for road sign recognition"IET Computer Vision Received on 31st March 2010

[16] J. Greenhalgh and M. Mirmehdi, "Real-time detection and recognition of road traffic signs," IEEE Trans. Intell. Transp. Syst., vol. 13, no. 4,pp. 1498-1506, Dec. 2012

[17] Wahyono, Kang-Hyun Jo ,"A Compa ative Study of Cla i fication Method fo T affic Sign Recognition"Inte national Confe ence on Industrial Technology (ICIT), Feb. 26 - Mar. 1, 2014, Busan, Korea

J. Wright, A. Y. Yang, A. Ganesh, S. Sastry, and Y. Ma. Robust face recognition via sparse representation. IEEE Transactions on Pattern Analysis and Machine Intelligence (PAMI), 31(2):210-227, 2008. 